\title{
Colorless Regenerative Amplification of Constant Envelope Phase-Modulated Optical Signals Based on Injection-Locked Fabry-Pérot Lasers
}

\author{
Alexandros Fragkos, Adonis Bogris, Dimitris Syvridis, and Richard Phelan
}

\begin{abstract}
An efficient phase preserving amplitude noise limiter suitable for constant envelope phase-modulated signals is experimentally demonstrated for $10-\mathbf{G b} / \mathbf{s}$ differential phase-shift-keying (DPSK) signals exploiting injection locking in Fabry-Pérot lasers. The limiter operates successfully over a 16.4-nm tuning range leading to $12 \mathrm{~dB}$ of power penalty reduction for $10^{-3}$ of bit-error-rate (BER) performance.
\end{abstract}

Index Terms - Injection-locked amplifiers, laser applications, limiting, noise and optical signal processing, phase modulation.

\section{INTRODUCTION}

$\mathbf{T}$ HE constantly increasing need for higher bit rates and longer optical transmission paths has led to alternative, more resilient to fiber losses and bandwidth efficient, modulation formats which exploit the phase of the optical field. Phase modulated signals exhibit a higher receiver sensitivity over conventional intensity modulation systems and suitability for multilevel signaling. The major impairment for phase modulation formats is the nonlinear phase noise produced by the accumulated amplitude noise of optical amplifiers and transferred to signal phase through fiber nonlinear effects, such as self-phase modulation (SPM) and cross-phase modulation (XPM). Till now, several techniques have been proposed for all-optical nonlinear phase noise suppression that are summarized in two basic categories; a) phase preserving amplitude limiters and b) phase sensitive parametric amplifiers.

Phase preserving amplitude limiters are active units that take advantage of nonlinear phenomena (Kerr effect), which take

\footnotetext{
Manuscript received July 22, 2011; revised September 13, 2011; accepted October 07, 2011. Date of publication October 17, 2011; date of current version December 09, 2011. This work was supported in part by the European Commission STREP project PHASORS (FP7-ICT-2007-2 22457) and in part by the European Union (Europian Social Fund-ESF) and Greek national funds through the Operational Program "Education and Lifelong Learning" of Funding Program: Heracleitus II. Investing in knowledge society through the European Social Fund.

A. Fragkos and D. Syvridis are with the Optical Communications Laboratory, Department of Informatics and Telecommunications, National and Kapodistrian University of Athens, Panepistimiopolis, Illisia, Athens, GR-15784, Greece.

A. Bogris is with the Departments of Informatics, Technological Educational Institute of Athens, Aghiou Spiridonos, 12210, Egaleo, Athens, Greece, and also with the National and Kapodistrian University of Athens, Panepistimiopolis, Illisia, Athens, GR-15784, Greece.

R. Phelan is with Eblana Photonics, Trinity College, Dublin 2, Ireland

Color versions of one or more of the figures in this letter are available online at http://ieeexplore.ieee.org.

Digital Object Identifier 10.1109/LPT.2011.2171941
}

place in highly nonlinear fibers (HNLF), to prevent the nonlinear phase noise buildup by periodically limiting the accumulated amplitude noise. In this category two are the most known topologies, the nonlinear amplitude limiter, which is based on the saturated four-wave mixing (FWM) in a parametric amplifier [1] and the Nonlinear Amplifying Loop Mirror (NALM) [2], which is based on SPM.

Phase-sensitive parametric amplifiers are based on FWM process in HNLF and act directly at the phase of the degraded signal. As shown by R. Slavik et al. in [3], when the appropriate phase relation is satisfied for the interacting waves, the phase noise suppression is remarkable leading to noteworthy performance.

Although both techniques are very promising for phase regeneration of high bit rate signals, they require extra components such as optical amplifiers, optical filters and suitable enclosure and electronics for thermal and mechanical vibration isolation. All these increase the fabrication cost, the complexity and the volume of the end product. Moreover, the limiting amplification which relies on the third order nonlinear effects in fibers obeys the typical S-shaped transfer function of optical gates which is not capable of suppressing extreme amplitude fluctuations and could be accompanied by detrimental amplitude-to-phase noise transfer [1].

In principle, the possibility of efficiently processing the amplitude content of an optical wave over a wide bandwidth without affecting its phase modulation fidelity is not only valuable for the prevention of nonlinear phase noise buildup. In addition amplitude limiters could promote the spectrally efficient combination of orthogonal data streams at the amplitude and the phase of the optical field and enable optical signal processing for applications such as the switching of optically labeled signals [4]. Recently, our group demonstrated the potential of an alternative amplitude limiter based on optical injection locking in a discrete mode semiconductor laser that offers highly efficient amplitude quantization, compactness, simplicity and ability to reproduce the phase modulation bandwidth of the incoming constant envelope phase modulated signal (CE-PM) up to multi GHz frequency range [5]. In this letter the principle is extended utilizing a Fabry-Pérot (FP) laser showing its ability for single mode operation and optical phase modulation amplification under injection locking. The usage of FP laser alleviates the need for exact wavelength matching between the incoming signal and the injection locked laser allowing for the operation of the limiting amplifier over almost the entire $C$-band wavelength region. In this particular 


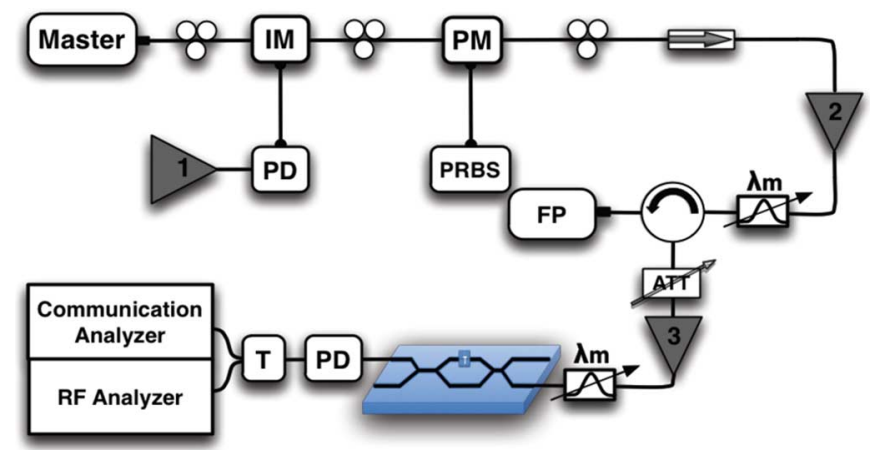

Fig. 1. Block diagram of the experimental setup. PM: Phase modulator. IM: Intensity modulator. PRBS: Pseudorandom binary sequence. EDFA1: Amplitude noise controller. EDFA2: Injection level controller. ATT: Variable attenuator. EDFA3: Preamplifier. PD: Photodiode. T: RF Splitter.

experiment, the regenerative amplification of CE-PM using FP injection locked lasers is demonstrated for $10 \mathrm{~Gb} / \mathrm{s}$ binary phase modulated signals over a $16.4 \mathrm{~nm}$ tuning range.

\section{EXPERIMENTAL RESULTS AND Discussion}

The block diagram of the experimental setup is depicted in Fig. 1. A tunable external cavity laser is used as the master laser and is phase modulated with a $2^{31}-1$ pseudorandom bit sequence (PRBS) at $10 \mathrm{~Gb} / \mathrm{s}$ using a $\mathrm{LiNbO}_{3}$ phase modulator. In this work, the case of a signal impaired at the amplitude is only studied. Such a signal could be produced at the output of a phase sensitive regenerator operating in the linear regime [3]. The intensity modulator (IM) is used to introduce noise only at the amplitude of master laser by modulating its field with the amplified spontaneous emission noise (ASE) produced by the first erbium doped fiber amplifier (EDFA), which is then converted to electrical noise via a $12 \mathrm{GHz}$ bandwidth photodiode. The two polarization controllers before the PM and the IM are used in order to align the laser with the modulation axis of each modulator. A second EDFA is placed just after the two modulation stages so as to compensate for the induced losses and control the power of the injected signal. The output of this amplifier is filtered, in order to remove ASE noise, with the use of an optical band-pass filter of $1 \mathrm{~nm}$ bandwidth and then is fed into the slave laser via a circulator. The output of the slave laser is sent to a variable attenuator and then amplified by a third EDFA which plays the role of a preamplifier to the receiver required for systematic bit-error rate (BER) measurements. The output of the master is also launched into the same amplifier in order to provide a fair comparison of the master and slave BER performances. Finally, the PSK signal, before (master) and after (slave) regeneration, is coupled into a 100 ps delay interferometer for the differential detection at a single $12 \mathrm{GHz}$ bandwidth photodiode and monitored in a $20 \mathrm{GHz}$ communication analyzer and a $26 \mathrm{GHz}$ spectrum analyzer.

In [4], we provided the first demonstration of the regenerative amplification of carrier-suppressed phase encoded signals with the use of injection locking. Successful limiting amplification requires that the slave laser is injection locked to the incoming signal.

Generally, the injection locking process in a multimode FP laser requires more optical power than in a single-mode laser

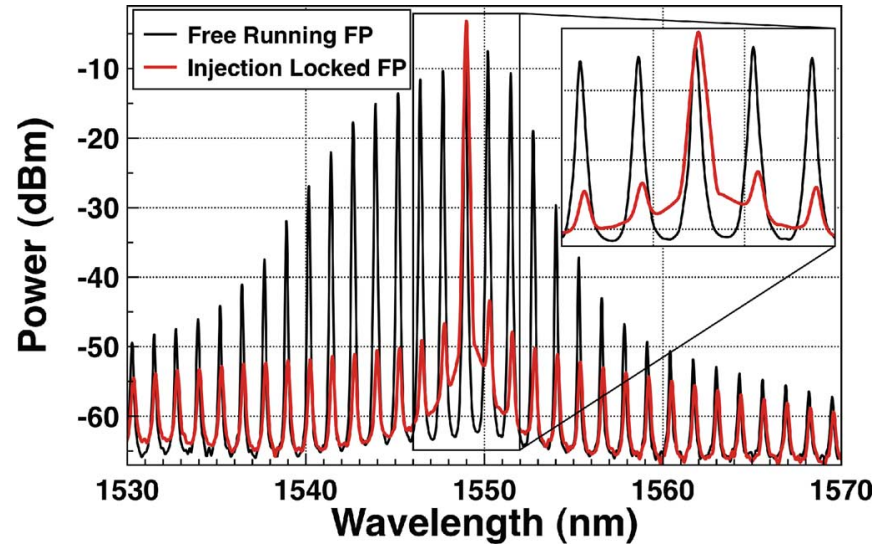

Fig. 2. Optical spectra of the free running and injection-locked FP laser.
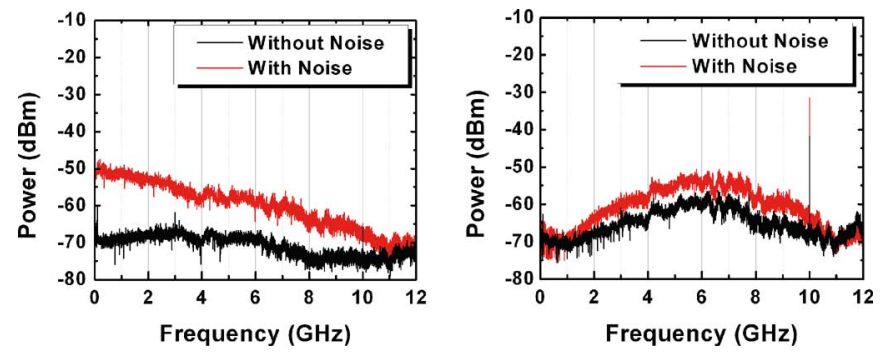

Fig. 3. Intensity noise spectra of the incoming signal (left figure) and the FP output (right figure).

because it is burdened with the additional requirement of suppressing the side modes [6]. So, to achieve single-mode operation, enhanced locking bandwidth and avoid transfer of the amplitude fluctuations of the incoming signal to the locked laser, the injected power needs careful adjustment.

Fig. 2 shows the optical spectra of the free running FP laser and the injection locked FP for an input wavelength of $1549.09 \mathrm{~nm}$ and slave operating current equal to $51.24 \mathrm{~mA}$.

The injected power measured at the input of the FP laser is $1 \mathrm{dBm}$ and the output FP power is equal to $8 \mathrm{dBm}$. This means that the requisite injection level for single mode operation and replication of the phase modulation is equal to $-7 \mathrm{~dB}$. Hence, the injection locked FP, operating as a limiting amplifier, provides a $7 \mathrm{~dB}$ fiber to fiber gain which should be scaled to more than $13 \mathrm{~dB}$ if coupling losses were taken into account. The locking properties were also examined at $1538.89 \mathrm{~nm}$ $(53.28 \mathrm{~mA})$ and $1555.29 \mathrm{~nm}(47.58 \mathrm{~mA})$, however the optical spectra are omitted due to space limitations. Through static experiments we observed that the locking bandwidth of the FP laser is $35.24 \mathrm{GHz}$ for the $1538.89 \mathrm{~nm}, 35.12 \mathrm{GHz}$ for the $1549.09 \mathrm{~nm}$ and $34.8 \mathrm{GHz}$ for the $1555.29 \mathrm{~nm}$ for an injection level of $-7 \mathrm{~dB}$. The locking bandwidth goes down to $10 \mathrm{GHz}$ for injection strength equal to $-15 \mathrm{~dB}$.

Fig. 3 shows the RF spectra of the incoming signal when impaired by ASE noise added at its amplitude and also the corresponding FP output. As it can be seen, the RF spectrum of the FP output exhibits a power enhancement around $6 \mathrm{GHz}$, which is attributed to amplitude relaxation oscillations occurring whenever the incoming signal phase changes phase state (from 0 to $\pi$ and vice versa) [7]. The phase jumps are equivalent to master 


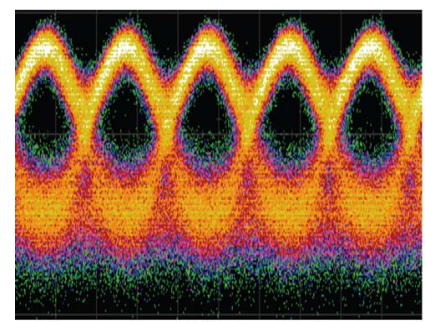

Master

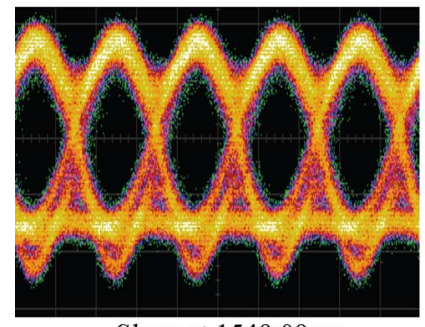

Slave at $1549.09 \mathrm{~nm}$

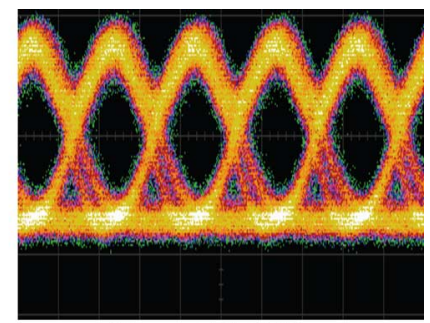

Slave at $1538.89 \mathrm{~nm}$

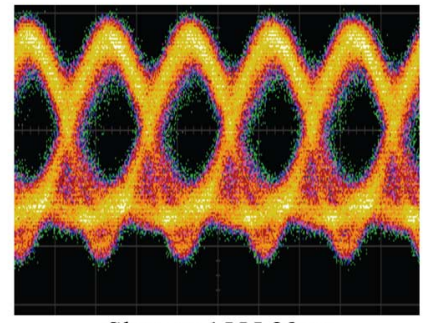

Slave at $1555.29 \mathrm{~nm}$
Fig. 4. 10-Gb/s DPSK eye diagrams of the input signal at $1538.89 \mathrm{~nm}$ degraded by ASE noise and the corresponding regenerated outputs at 1538.89, 1549.09, and $1555.29 \mathrm{~nm}$.

frequency shifts which temporarily unlock the slave and thus cause its relaxation oscillations. This phase-to-amplitude modulation conversion creates an NRZ-like RF spectrum with suppression at the lower frequencies due to the dc balanced amplitude oscillations. However this phase-to-amplitude conversion emerges at the bit transitions and hence is not expected to affect the eye opening of the DPSK signal.

In Fig. 4 , the eye diagrams of the degraded by amplitude noise input and the regenerated output differentially phase shift keying (DPSK) signal are depicted for three different wavelengths (1538.89 nm, $1549.09 \mathrm{~nm}$ and $1555.29 \mathrm{~nm})$. The figure shows clearly that FP laser is capable of suppressing the unwanted amplitude noise within a wavelength band of $16.4 \mathrm{~nm}$. Outside this region, FP laser is able to lock but the injection level needed for single-mode operation is extremely high to allow limiting amplification and noise suppression. The authors strongly believe that a further optimization of the experimental conditions in terms of the FP operating current could extend the operation bandwidth at least up to $20 \mathrm{~nm}$.

Fig. 5 shows a series of BER measurements conducted for input OSNR equal to $23 \mathrm{~dB}$, which further prove the remarkable amplitude limitation properties of the proposed injection locked laser amplifier. The amplitude noise level was adjusted appropriately so as the input signal to exhibit constant BER for every wavelength inside the investigated spectral range. The FP laser provides a reduction of $12 \mathrm{~dB}$ in the required receiving power for the achievement of BER performance equal to $10^{-3}$. Additional experiments which are not shown here, exhibited that the injection locked FP laser is also capable of improving the properties of signals degraded by ASE noise as well, analogously to what was presented in [5] for discrete-mode slave lasers.

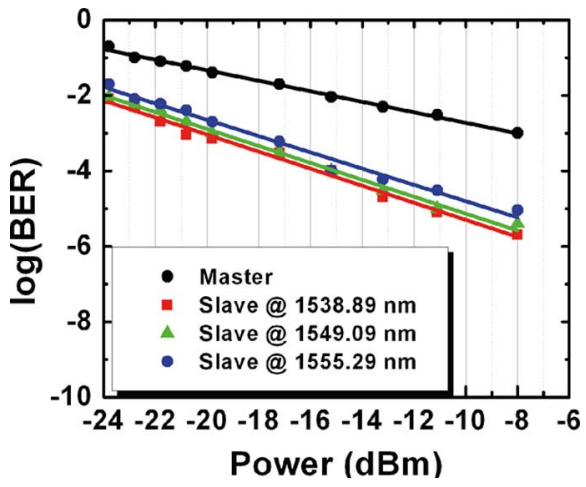

Fig. 5. BER performance as a function of the receiving power for degraded master signal (black trace) and the corresponding regenerated signal at the output of slave at $1538.89 \mathrm{~nm}$ (red trace), $1549.09 \mathrm{~nm}$ (green trace), and $1555.29 \mathrm{~nm}$ (blue trace).

\section{CONCLUSION}

An amplitude limiter able of treating input wavelengths within a $16.4 \mathrm{~nm}$ range was experimentally demonstrated exploiting the injection locking properties of FP lasers. The FP laser is capable of suppressing high levels of amplitude noise without affecting the fidelity of the phase content. The modulation bandwidth enhancement of the injection locked lasers, reaching up to $100 \mathrm{GHz}$ [8], in addition to the low cost of these devices and their potential for monolithic integration with other components paves the way for optical signal processing techniques which could enable bandwidth efficient and cost effective next generation access and metro optical networks.

\section{REFERENCES}

[1] M. Matsumoto and K. Sanuki, "Performance improvement of DPSK signal transmission by a phase-preserving amplitude limiter," Opt. Express, vol. 15, pp. 8094-8103, 2007.

[2] C. Stephan et al., "Nonlinear phase noise reduction in a DPSK transmission system using cascaded nonlinear amplifying loop mirrors," IEEE Photon. Technol. Lett., vol. 21, no. 24, pp. 1864-1866, Dec. 15, 2009.

[3] R. Slavik et al., "All-optical phase and amplitude regenerator for nextgeneration telecommunications systems," Nature Photon., vol. 4, pp. 690-695, 2010.

[4] X. Liu et al., "Transmission of an ASK-labeled RZ-DPSK signal and label erasure using a saturated SOA," IEEE Photon. Technol. Lett., vol. 16, no. 6, pp. 1594-1596, Jun. 2004.

[5] A. Fragkos, A. Bogris, D. Syvridis, R. Phelan, J. O'Carroll, B Kelly, and J. O'Gorman, "Amplitude regeneration of phase encoded signals using injection locking in semiconductor lasers," in Proc. OFC/NFOEC, Los Angeles, CA, 2011.

[6] N. H. Zhu et al., "Single mode operation of a Fabry-Perot laser locked by a tunable laser," Microw. Opt. Technol. Lett., vol. 50, no. 7, pp. 1888-1892, 2008.

[7] Y. Awaji, T. Kuri, W. Chujo, M. Naganuman, and K. Kitayama, "Differential-phase-to-intensity conversion based on injection locking of a semiconductor laser," Opt. Lett., vol. 26, pp. 1538-1540, 2001.

[8] E. K. Lau, H.-K. Sung, and M. C. Wu, "Frequency response enhancement of optical injection-locked lasers," IEEE J. Quantum Electron., vol. 44, no. 1, pp. 90-99, Jan. 2008. 